\title{
Neural Networks for Combined Control of Capacitor Banks and Voltage Regulators in Distribution Systems
}

(Summary)

2. $\mathrm{Gu}$

Oak Ridge National Laboratory, Oak Ridge. TN 37831
D. T. Rizy

\author{
BH:
}

FEB 05 19S3

OSTI

Conventional model based analysis and computing methods are not adequate for real time control of present and future distribution systems because of their complexity, nonlinear nature, and size. On the other hand, artificial neural networks (NN), which emulate the structures of biological nervous systems, have many properties that make them suitable for real-time power system applications. The development and application of neural networks for combined control of capacitor banks and voltage regulators in a distribution system is described. The control is optimal in the sense of minimizing system energy ( $\left.I^{2} R\right)$ loss with the constraint condition of maintaining all bus voltages within standard limits $( \pm 5 \%)$. Given the loading of the distribution systems, the problem is to determine the tap or switch states of the capacitor banks and voltage regulators such that the cumulative $I^{2} R$ loss of the whole distribution system is minimized without violating standard or specified voltage limits.

The general principles of neural network design for the specific problem are described. While the number and values for the outputs of the neural net is decided by the controllable devices, it is important to select the input variables that contain enough information to solve the problem. A three- laver architecture for the neural net controller is adopted because previous studies have shown that this structure is sufficient for generalization. It is also important to select the training data that reasonably covers the whole input-output spaces of system operation. Finally, the local minimum problem for the back propagation algorithms should be avoided during the training process. Unfortunately, there exist no systematic methods that guarantee a global solution.

A neural net controller for a radially configured distribution system with 30 buses, 5 switchable capacitor banks and one 9 tap voltage regulator is designed based on the above principles. For comparison, two different input sets are used. The first input set uses $P, Q$ and $V$ from 6 measurement points in the distribution circuit. The second set added the 6 output variables as its input in addition to the original input variables thus providing feedback to the neural network. The hidden layer has 13 nodes after a trial and error process. The training data is obtained by equally dividing the maximum load into 13 levels, and then solving the optimal problem with an exhaustive search. Since the 13 pairs of $1 / O$ data can not train the neural net satisfactorily, a data expansion approach that expands the 13 pairs of data to 3744 pairs without increasing the computational complex was introduced. This is based on the fact that for a given load level, there is only one optimal solution corresponding to all possible tap states of controlled devices. Monte Carlo simulation technique is used to test the neural controller. Five different load patterns ranging from a smooth sinusoidal curve to a purely random sequence uniformly distributed in $(0,1)$, and four levels $(0 \%, 25 \%, 35 \%$ and $50 \%)$ of load distribution deviation on each bus are considered in the simulation. The simulation is executed on 12,000 samples over all the 20 test cases. For comparison, the true solutions for the 20 cases are also calculated over a smaller sample size. The results show that energy losses for the neural controller is not significantly larger than the true solution's, and there are some occurrences of voltage out of bounds when the load and distribution deviation is large. However, the frequency of this occurrence and the values of out bound voltages are not very significant as shown by the statistical data. The simulation also shows that the neural controller with feedback can outperform the one without feedback.

In summary, with fewer training data obtained from the optimization process, combined with the training data expansion approach, we built a relatively good performing NN controller that otherwise would fail if using the original training data set only. The results show that, proper selection of the input variables and training set that covers the input/output space of the system being modeled is important in building a successful neural network.

DISTRIBUTION OF TISS LOOUREIT IS UNUMATED 饮 


\title{
NEURAL NETWORKS FOR COMBINED CONTROL OF CAPACITOR BANKS AND VOLTAGE REGULATORS IN DISTRIBUTION SYSTEMS
}

\author{
Z. Gu, Member $\quad$ D. T. Rizy, Senior Member
}

\author{
Oak Ridge National Laboratory, Oak Ridge, Tennessee
}

\begin{abstract}
A neural network for controlling shunt capacitor banks and feeder voltage regulators in electric distribution systems is presented. The objective of the neural controller is to minimize total $I^{2} R$ losses and maintain all bus voltages within standard limits. The performance of the neural network for different input selections and training data is discussed and compared. Two different input selections are tried, one using the previous control states of the capacitors and regulator along with measured line flows and voltage which is equivalent to having feedback and the other with measured line flows and voltage without previous control settings. The results indicate that the neural net controller with feedback can outperform the one without. Also, proper selection of a training data set that adequately covers the operating space of the distribution system is important for achieving satisfactory performance with the neural controller. The neural controller is tested on a radially configured distribution system with 30 buses, 5 switchable capacitor banks and one nine tap line regulator to demonstrate the performance characteristics associated with these principles. Monte Carlo simulations show that a carefully designed and relatively compact neural network with a small but carefully developed training set can perform quite well under slight and extreme variation of loading conditions.
\end{abstract}

KEYWORDS: neural networks, artificial intelligence, real-time power systems control, capacitor control, regulator control, distribution automation.

\section{INTRODUCTION}

Changes in the utility industry including increasing deregulation and competition, integration of a more diverse portfolio of supply and demand resources, financial and environmental constraints of building new construction is adding significant complexities to the operation of electric power distribution. More sophisticated real time control systems are needed for distribution systems to provide electric power with higher efficiency, quality, and security without the need for building new facilities. In addition to traditional control such as voltage/reactive power (var) control, the increasing use of new power generation, storage, and conversion technology, as well as the incorporation of independent power dispatches, further increases the difficulty of implementing such real time control systems. Conventional model based analysis and computing methods are not adequate for real time automation of present and future distribution systems because of their complexity, nonlinear nature, and size. On the other hand, artificial neural networks (NN), which emulate the structures of biological nervous systems, have many properties that make them suitable for real-time power system applications, such as real time analysis, estimation, prediction and control. These properties include "model free" input-output mapping, inherited non linearity, massively parallel and distributed connections of simple processor units, and adaptability to environmental changing.

In distribution system operation, shunt capacitor banks and feeder regulators are necessary for providing acceptable voltage profiles to all end-use customers and reducing energy losses associated with power line on large distribution systems. These capacitors and regulators need to be controlled according to different load patterns, otherwise they do not meet their design objective and can indeed cause voltage problems and increased losses.

Conventional optimization methods, such as dynamic programming (DP), for determining capacitor switch
The submitted manuscript has been authored by a contractor of the U.S. Government under contract No. DE-AC05-84OR2 1400. Accordingly, the U.S. Government retains a nonexclusive. royalty-free license to publish or reproduce the published form of this contribution, or allow others to do so, for U.S. Government purposes. 
operation and regulator tap positions are time consuming and impossible for large systems. It is unrealistic to directly apply these methods to real time optimal control of distribution systems. Because a voltage regulator generally has many tap positions (typically 32 providing $5 / 8$ with each tap), the combined voltage regulator and capacitor banks control is more complex and difficult.

Although there are many papers published on NNs applied to power systems. (part of them can be found in a survey in [1],) few papers can be found for combined control of capacitor banks and voltage regulators in distribution systems. Some papers have been published for NN control for capacitors only without considering the voltage regulators $[2,3]$. In this paper, we present an experimental neural network controller for control of the capacitor banks and tap positions of feeder regulators. The neural controller is applied to a 30 bus radial distribution system with five switchable capacitors and one voltage regulator with 9 taps. The optimization of this combined capacitor bank and voltage regulator control is to minimize the energy loss of the whole distribution circuit under the constraint condition that bus voltage remain within standard limits (within $\pm 5 \%$ of nominal).

In section 2, the combined capacitor and regulator control problem is presented. Section 3 describes the design process of the NN controller. In section 4, the simulation of the neural controller for the 30 bus distribution system is given. Finally, section 5 gives conclusions.

\section{PROBLEM DESCRIPTION}

In this study, two assumptions are made in regards to the simulation of the combined capacitor and regulator control. First, the locations and ratings of the capacitors and voltage regulators are assumed to be given and fixed for the system to be controlled. Second, it is assumed that instrumentation and communication is in place for simultaneous collecting (or synchronizing) real time measured line flows and bus voltages at various locations on the distribution system. The unit of time mentioned in this study could refer to any segments of time such as 1 sec., 1 minute, 1 hour, etc.. The loads are modeled as constant complex power which do not exceed the designed maximum values.

Given the loading of the distribution systems, the problem is to determine the on/off states of the capacitors (or tap settings for adjustable capacitor banks) and the tap settings of the voltage regulators so that the cumulative $I^{2} R$ loss of the whole distribution system is minimized without violating standard or speci-

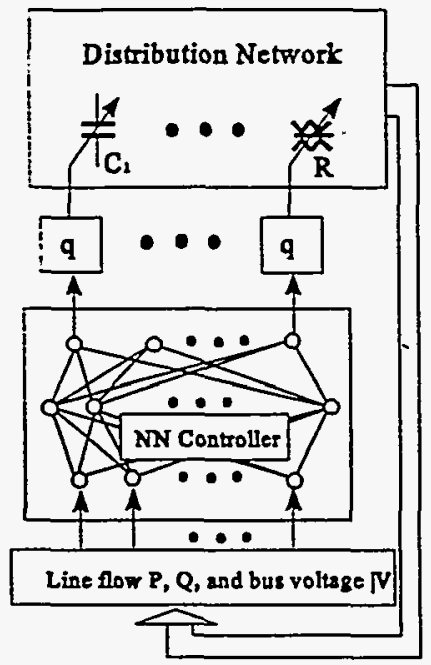

Figure 1: The system structure of the NN controller for the distribution system.

fied voltage limits.

This can be shown mathematically as follows:

$$
\begin{array}{rc}
\operatorname{argmin} & {\left[\sum_{i=1}^{M}\left|I_{i}(\mathrm{~T})\right|^{2} R_{i}\right]_{(P(t), Q(t))}} \\
\text { s.t. } & V_{j} \in\left(V_{j \_ \text {min }}, V_{j \_ \text {max }}\right), \quad j=1, \ldots, N .
\end{array}
$$

where $M$ and $N$ are the total number of lines and buses respectively, $V_{j \_ \text {min }}$ and $V_{j \_ \text {max }}$ are the lower and upper voltage limit of each bus, and $T$ is the $L$ dimensional tap position vector for the total $L$ number of capacitor banks and voltage regulators in the circuit. Note that when the active power, $P$ and reactive power, $Q$ of each bus are given, the complex line current $I_{i}$ is only a function of $T$. The voltage constraint followed in this study is to maintain the lower and upper voltages for each bus within $\pm 5 \%$ p.u.

Thus, for a given set of loads at time $t$ at each bus, we want to find the optimal tap position vector $T$ that minimizes the $I^{2} R$ loss of the whole distribution circuit. This is a nonlinear combinatorial optimization problem. It can not be solved in real time using traditional methods such as power flow analysis and dynamic programming if the number of capacitors and regulators and their taps are large. As mentioned in Section 1, a trained neural network provides a computationally efficient way to solve the problem in real time although the training process may take a long time and is generally carried out off line.

\section{THE DESIGN OF THE NEURAL NETWORK CONTROLLER}

There are numerous neural network approaches with different structures, transfer functions, and training al- 
gorithms. While not every problem can be solved via $\mathrm{NN}$, some problems may be solved by several different NN approaches. In this paper, we adopt an NN approach with a three-layer feed forward structure and the back propagation algorithm because of its relative simplicity: maturity in regards to applications in different fields. and common use. More importantly, previous studies [4] have shown that this structure is sufficient for generalization.

The structure of the neural controller for the distribution system is shown in Figure 1. The inputs to the neural controller are the measured line flows $P$ and $Q$ and bus voltage magnitudes $V$ at select points on the distribution circuit. The input and associated outputs for the selected points and states of operation of the distribution system should contain enough information needed to solve the optimization problem (1). However, how many measurement points are needed, where should the locations of these points be, and which circuit parameters $(P, Q$ and/or $V)$ should be used inputs to the N.I remain open questions. The answers to these questions have practical significance because the fewer the number of measurement points, the lower the cost of the control system. Intuitively, the selection of these measurement points should be close to the locations of controllable devices. The selection of the input variables could be more complex if the subject distribution system has a network structure.

The number of outputs of the NN are determined by the number of capacitor banks and voltage regulators to be controlled and their tap states. One NN output was assigned for each device in order to reduce the number of $\mathrm{YN}$ outputs. Since the transfer function at the output layer of the $\mathrm{NN}$ is a hyperbolic tangent, the output of the neuron active function is a continuous value. Thus, each controllable device which has multiple (or binary) state needs a multiple (or binary) level quantizer $\mathbf{q}$ after each $\mathrm{NN}$ output.

In regards to the $\mathrm{NN}$ architecture, it is important to determine the number of hidden layers and the number of nodes on each hidden layer. One way to build the structure is based on the "growing idea" $[5,6,7]$. There are also some "rules of thumb" or formulas to find the number of nodes of the hidden layer based on the input number. However, if there is little or no $a$ prior knowledge about the problem, the best structure can only be obtained by trial and error.

The training process is usually carried out off line because of the inefficiency of existing training algorithms, although it is possible to adapt the neural network on line as new data comes in. A set of known input and output data that satisfy the problem (1) are needed to train the NN controller, where the input data is the measured $P, Q$ and/or $V$ at some measurement points, and the output is the optimal switching states (tap positions in the case of adjustable) of capacitor banks and tap positions of voltage regulators corresponding to some given load pattern of the distribution system. Because neural networks are in principle generalizers or interpolators for a given set of training data, the performance of the trained neural network is dependent, to a large degree, on the selection of the training data. Thus, the training input data should reasonably cover the whole input space under possible operational conditions, and it is important to carefully select or design the training data. Some auxiliary technique such as simulation may be needed when it is necessary and possible.

The back propagation training algorithm requires setting of its learning rate, and in some cases the momentum in the training process. Some adaptive and fuzzy logic approaches $[8,9]$ may be used to decide these parameters dynamically. However, the steepest descent algorithm may fall into undesired local minimum solutions making achieving a global optimal solution difficult. The problem can be caused by not selecting a proper initial setting of the weights and bias. However, no systematic method exists to find an initial setting that guarantees a global optimal solution.

After building the neural network and training it, a test process must be carried out. The test process should use those I/O pairs that are not used in the training process. Only after the test results are satisfied can the NN controller be applied to the real time systems.

\section{SIMULATION RESULTS}

A NN controller that applies the design methods described in the previous section was applied to a 30 bus test distribution system described in [10,2]. However, the ratings of the capacitor banks given in [2] were increased and a feeder voltage regulator was added at bus 4 so that the voltage constraint conditions could be met for maxium loading. The voltage regulator is modeled with 9 tap positions which are distributed equally between ratio 1 and 1.1 p.u.. Even though the technique described above is capable of controlling adjustable capacitors (with multiple tap settings) only conventional two-state capacitors (on/off) are studied in this paper while [2] considered 4 tap positions for all the capacitors. The new ratings of the capacitors are shown in Tabel 1.

Figure 2. shows a 3-D one line diagram of the 30bus distribution system. The vertical bars indicate the maximum active power loads on each bus. The reactive 


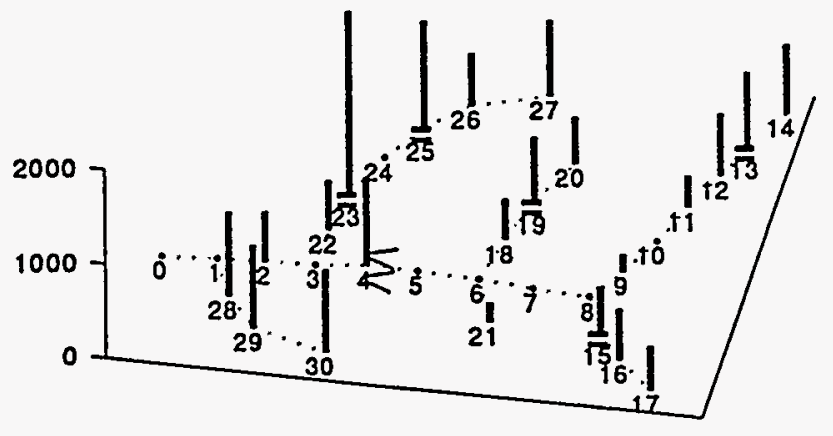

Figure 2: One-line diagram of the 30-bus Distribution System. The vertical bars are the maximum active powers in $\mathrm{KW}$. The 6 measurement points are at the up stream sides of Bus 1, Bus 22, Bus 4, Bus 8, Bus 9 and Bus 19.

power loads are fixed as one third of active power as in $[10,2]$.

Tabel 1. Capacitor ratings for the test system.

\begin{tabular}{|l|l|l|l|l|}
\hline \multicolumn{5}{|c|}{ Capacitor Ratings (KVAR) } \\
\hline \hline$C_{13}$ & $C_{15}$ & $C_{19}$ & $C_{23}$ & $C_{25}$ \\
\hline 800 & 1000 & 400 & 1600 & 2000 \\
\hline \hline
\end{tabular}

Thus, by the design principle in Section 3, the NN controller has 6 outputs. The five outputs for capacitors are fed into 5 binary quantizers and the output for the voltage regulator is fed into a 9 level quantizer. The training output data for the capacitors and regulator are simply the tap position index $\{0,1\}$ and $\{0$, ..., 8\} respectively.

Two different input selections for the NN are studied. For comparison, the first set of input variables are chosen the same as in [2]. ${ }^{1}$ This first set of 24 inputs consists of the power flows $P$ and $Q$ and voltage magnitudes $V$ on the 6 measurement points shown in Figure 2, which are obtained by solving the power flow equations for the system, and the current tap positions of the capacitors and regulator. The second set tested another NN controller without the tap positions as its input since inputs $P, Q$ and $V$ should contain some information about the current tap positions. The performance of the two controllers was compared.

The structure of the NN controller that was selected has only one hidden layer with 13 nodes. (This

\footnotetext{
${ }^{1}$ Several different input variable sets were tried without significantly altering the training error.
}

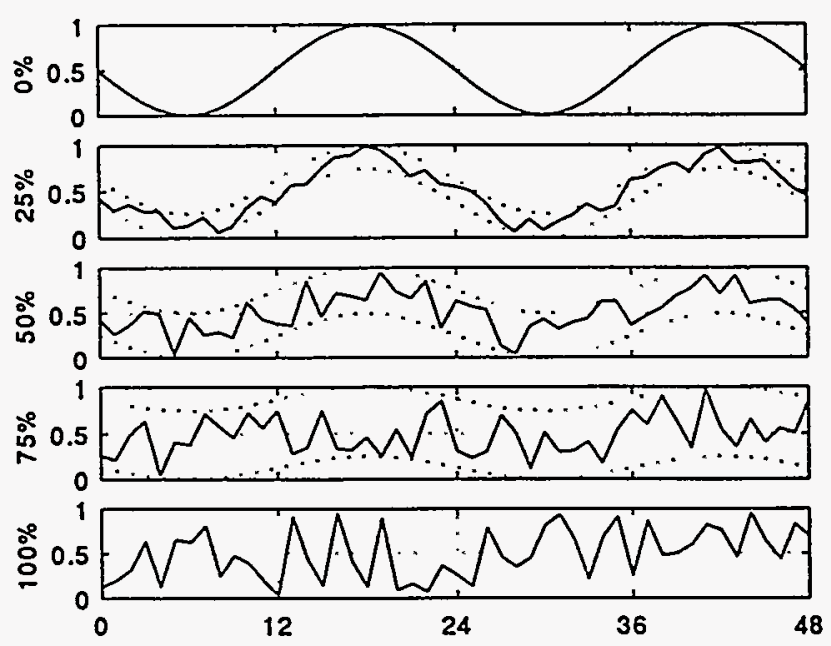

Figure 3: Five different degrees of load profile deviations over two sine periods.

structure is much smaller than the one in [2] where two stages of 3 layer NN are used and binary outputs are used to represent multi tap positios.) The neuron transfer functions on the hidden and output layer are chosen as hyperbolic tangent.

Although the input variables are selected as the same as in [2], the training data are collected in a different way. In order to cover the whole input space, the training data are selected by uniformly dividing each bus load from zero to maximum into 12 segments. Thus, the 13 load levels are used to obtain the input $P, Q$ and $V$ data and the corresponding output tap positions of the capacitors and the regulator, which are obtained by solving problem (1) 13 times.

The testing of the NN controller included conducting Monte Carlo simulations to try to replicate the conditions under which NN would bave to operate in the field. Two variations, the total load level and the load distribution patterns, are simultaneously considered. In one dimension, the total load level is varied from a smooth sinusoidal curve to a noisy one with different noise magnitude to simulate real-time power system data. The noise is uniformly distributed in $(0,1)$. Figure 3 shows the 5 different load curves during two periods, where $0 \%, 25 \%, 50 \%, 75 \%$ and $100 \%$ correspond to the magnitudes of added noise. Note in the $100 \%$ variation case, it is possible for the load level to change from maximum load to zero load or vise versa in one time increment.

The load distribution patterns of the buses may also vary randomly. That is, the load demand on each individual bus may deviate $\pm x(t) \%$ independently both with respect to space and time, where $x(t)$ is a uni- 
formly distributed random variable in four different intervals, $(0,0),(0,25),(0,35)$, and $(0,50)$. The resultant load Distribution Pattern Deviation (DPD) is normalized so that the sumation of the total load of the whole circuit would not exceed the total load level.

Thus, there are a total of 20 test cases ( 5 loading cases times 4 demand cases) to introduce different variations on both loading level and distribution patterns. The purpose of this design is to test the robustness of the neural network controller and compare different approaches. In order to get sufficiently accurate statistical data, each Monte Carlo run is executed through 12,000 units of sampling time (represents 500 days if a unit is 1 hour or 8.33 days if a unit is 1 minute).

We first used the $13 \mathrm{I} / \mathrm{O}$ data pairs to train the neural network. Each input data vector is composed of the $18 P, Q$ and $V$ values obtained from the 6 measurement points. Each output data vector is composed of 6 corresponding tap states of the 6 controlled devices. Although the recall error ${ }^{2}$ is very small, the trained neural network can not pass the simulation test even without any variations. The results show that the bus voltages at about half the buses will be out of the specified limits of .95 to 1.05 more than $50 \%$ of the time.

The above results indicate that the $13 \mathrm{I} / \mathrm{O}$ training data pairs can not train the neural network to be sufficiently general for all possible states in the I/O spaces. More training data, or perhaps more complex neural net structures, are needed. We know for a given load level, the optimal solution of (1) is obtained from all possible $288=2^{5} \times 9$ state vectors of the 5 switchable capacitors and one 9 tap voltage regulator. That means for the same load level, the neural network should map all the possible 288 input state vectors, which are corresponding to the 288 possible tap positions of the capacitors and the regulator, onto the very same optimal tap position vector only. Thus, for each load level, we may have 288 pairs of $\mathrm{I} / \mathrm{O}$ training data which are obtained only for solving (1) once. Accordingly, we finally obtained $3744=288 \times 13$ pairs of training data without increasing the computational cost. By this approach of collecting training data, the number of expansive optimization processes can be reduced significantly.

The above training data expansion approach also significantly improves the NN controller's performance. As mentioned earlier, we have two neural network approaches for solving the same problem. Approach A only takes the $18 P, Q$ and $V$ from the 6 measurement points as the elements of the input vector. Approach $B$ adds the 6 tap positions in addition to the original 18 elements, which is the same way of composing the

\footnotetext{
${ }^{2}$ Recall error is the error between the actual or desired output and NN output by applying the training data only.
}
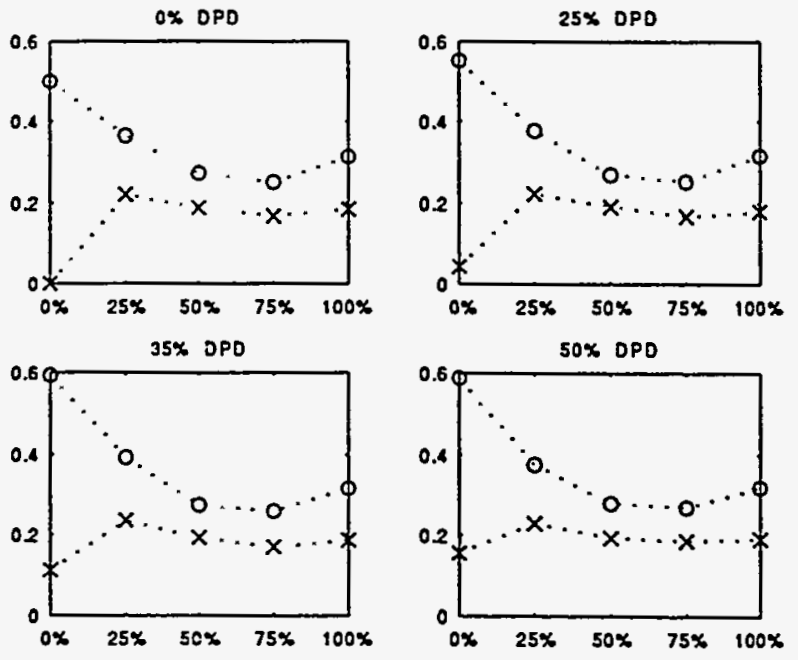

Figure 4: The frequency of over limit occurrence. o: approach $A$. $x$ : approach $B$.
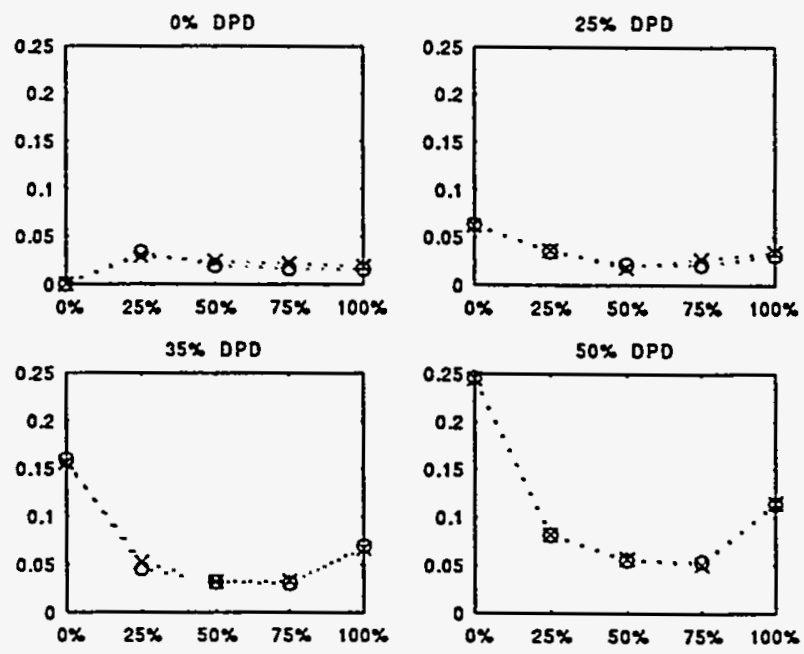

Figure 5: The frequency of under limit occurrence. o: approach $\mathrm{A}, \mathrm{x}$ : approach $\mathrm{B}$.

input vector as in [2]. B can also be considered as introducing feedback, which can generally improve control accuracy as well known in control engineering. Thus $B$ outperforms $A$ which is not unexpected.

In comparing the results of the two approaches, we find that the energy losses for approach $B$ during the whole simulation period ranges from $3,618 \mathrm{MWH}$ to 4,645 MWH among the 20 test cases. The energy loss of approach $A$ is very close to $B$ with a maximum of $.09 \%$ larger than $B$. Since it is too time consuming to obtain the true solutions for all the 12,000 samples of all the 20 cases, we used the true optimal solution for the first 49 samples of all the 20 cases (thus a total of 980 samples) for comparision. The energy losses of both approaches are very close to the true solutions' energy losses, which 

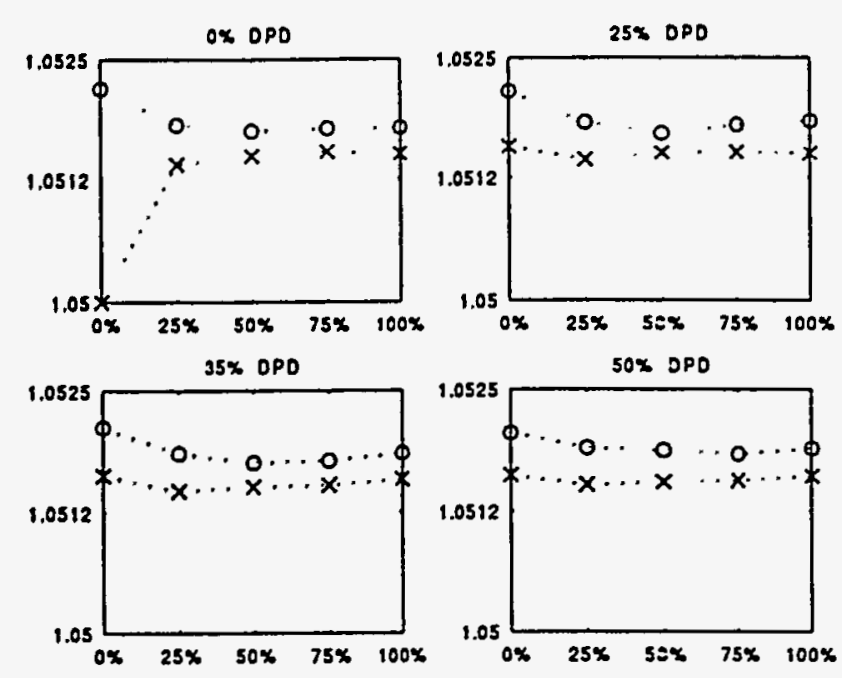

Figure 6: The average over limit voltage. o: approach $A, \mathrm{x}$ : approach $\mathrm{B}$.
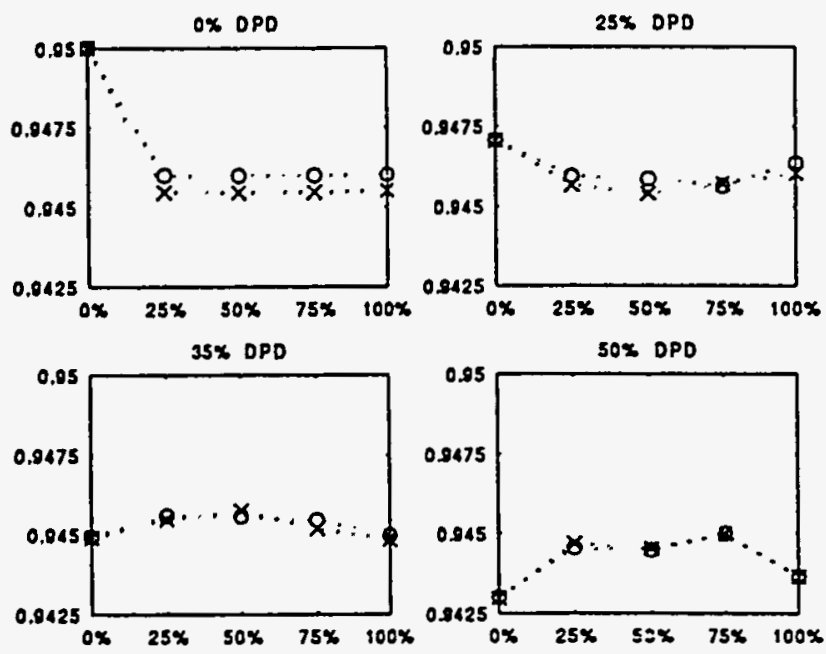

Figure 7: The average under limit voltage. o: approach $A, x$ : approach $B$.

range from $12.65 \mathrm{MWH}$ to $18.64 \mathrm{MWH}$ for all the 20 cases. The average energy losses of approach $\mathrm{A}$ and $\mathrm{B}$ are about $0.19 \%$ and $0.13 \%$ higher than the true energy losses respectively. Note among the 980 samples, 13 are excluded because no true optimal solution exists for these severely varied load and distribution patterns.

Geometrically, the convex hull of the feasible solution set of a combinatorial optimization problem with constraint conditions is a polytope, which can be composed by a finite number of hyperplanes. On the other hand, the boundary of the output set of a neural network is generally a closed hypercurve if continuous sigmoidal transfer functions are used. Therefore, there will be some output points of the neural network lying outside of the feasible solution set, which means some solutions given by the neural network may not meet the constraint conditions. This result is directly from the inherited characteristic of neural networks: its ability to generalize the whole I/O space mapping with only partially available training data.

In some applications, if the infeasible solutions given by the neural networks do not violate the constraint conditions too much. and if they do not occupy a large part of the output space of the neural netrorks, then these solutions may still be accepted. This is a power quality issue in terms of our application. We thus studied the frequency of the occurrence of out-of-limit voltages and the magnitude of these voltages in the simulation.

Figure 4 and Figure 5 show the frequency of over and under voltage limit occurrences for the 20 test cases, where DPD stands for Distribution Pattern Deviation. The frequency is obtained by adding the numbers of all buses whose voltages are under (or over) the limit, and then dividing the sum by the number of total time units (12.000). Thus the vertical axis indicates the number of buses that are either over or under the voltage limit per time unit. Clearly, for the over limit occurrence, approach B has a lower frequency of outof-range voltages for all 20 cases but about the same as approach $A$ for the under limit occurrences. We can also see that the frequency of over limit occurrences is higher than under limit occurrences, and the NN controller is sensitive to the DPD for the under limit cases, i.e., the greater the DPD, the higher the under limit frequency. It was found that most of the over limit voltages occur on bus 5 that does not have load connected.

Figure 6 and Figure 7 show the average values for these out-of-limit voltages. The statistical data show the similar rsults for the average out-of-limit voltages as the out-of-limit frequency results. Notice that the out-of-limit voltages do not exceed the limits by very much on average (less than .0025 higher or .0075 lower than the voltage limits). Figure 8 shows the voltages for buses 5 and 27 at which most over and under voltages occur, where LDD stands for LoaD Deviation. (Note in the 50\% DPD and $100 \%$ LDD case, there is a point at which no true solution exists.)

\section{CONCLUSION}

Some occurrence of infeasible solutions can result from neural networks when solving optimization problems with constraint conditions due to the neural network's generalization or interpolation property. However, the Monte Carlo simulation of the 30-bus test distribution system over a large sample size and 20 different vari- 


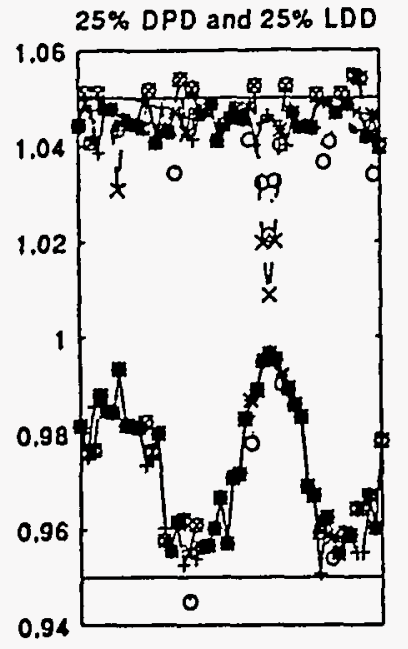

50\% DPD and 100\% LDD

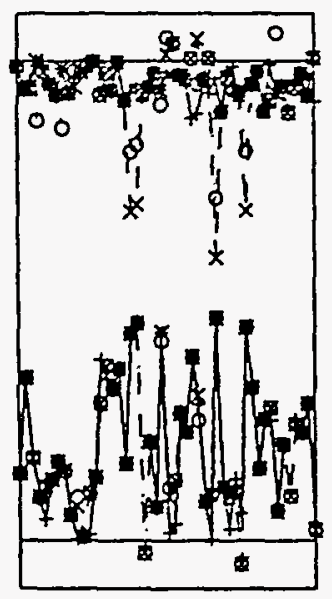

Figure 8: The voltage profiles at Bus 5 and Bus $2 \vec{\jmath}$ over a two sine periods. o and dot line: approach $A . x$ and dash line: approach B. + and solid line: true optimal solution.

ation cases indicates that this frequency may not be high, and the average magnitudes of these output deviations from desired or specified values may not be significant if the neural network is carefully designed. If no infeasible solutions are allowable at all, some extra measures need to be taken. One possible approach may be to apply more strict voltage limitation to obtain the training data though it seems too conservative.

With fewer training data obtained from the optimization process, combined with the training data expansion approach, we built a relatively good performing NN controller that otherwise would fail if using the original training data set only. The results show that, proper selection of the training set that covers the input/output space of the system being modeled is important in building a successful neural network. The comparison of the two input data design approaches also indicates that neural networks with feedback can generally outperform the one without feedback.

\section{ACKNOWLEDGEMENT}

The research described herein was sponsored by the Office of Energy Management, U.S. Department of Energy, under Contract No. DE-AC05-84OR21400 with Lockheed Martin Energy Systems. It was also supported in part by an appointment to the Oak Ridge National Laboratory Postdoctoral Research Associates Program administrated jointly by the Oak Ridge National Laboratory and the Oak Ridge Institute for Science and Education.

\section{REFERENCES}

[1] V. S. S. Vankayala and N. D. Rao, "Artificial neural networks and their applications to power systems - a bibliographical survey;" Electric Pouer Systems Research, vol. 28, 1993, pp. 6i-i9.

[2] N. I. Santoso and O. T. Tan, "Neural-net based real-time control of capacitors installed on distribution systems," IEEE Trans. Power Delivery, vol. 5, no. 1, Jan. 1990, pp. 266-72.

[3] Y. Y. Hsu and C.C. Yang, "A hybrid artificial neural network-dynamic programming approach for feeder capacitor scheduling," IEEE Trans. Power systems, vol. 9, no. 2, May, 1994, pp. 1069-75.

[4] K. Hornik, M. Stinchcombe and H. White, "Multilayered feedforward neural networks are universal approximators, "Neural Networks, vol. 2, 1989, pp. 359-366.

[5] Z. Gu and WV. A. Sethares, "Structural adaptation of neural networks for logic functions," Proc. Conf. Information Science and Systems, Baltimore, MD, May 1989, pp. 578-83.

[6] S. E. Fahlman and C. Lebiere, "The cascadecorrelation learning architecture," Tech. Rep. No. CMU-CS-90-100, School of Computer Science, Carnegie-Mellon Univ., Pittsburgh, PA, 1991.

[7] T. C. Lee. A. M. Peterson and J. C. Tsai, "A multi-layer feed-forward neural network with dyanmically adjustable structures," IEEE Intl. Conf. Systems, Man, and Cybernetics, Los Angeles, CA, 1990, pp. 367-69.

[8] J. J. Choi, P. Arabshahi, R. J. Marks II and T. P. Caudell, "Fuzzy parameter adaptation in neural systems," Intl. Joint Conf. on Neural Networks. vol. 1, Baltimore, MD, 1992, pp. 232-38.

[9] R. A. Jacobs, "Increased rates of convergence through learning rate adaptation," Neural Networks, vol. 1, 1988, pp. 295-307.

[10] J. J. Granger and S. Civanlar, "Volt/Var control on distribution systems with lateral branches using shunt capacitors and voltage regulators," IEEE Trans. Pouver Apparatus and Systems, vol. PAS-104, no. 11, Nov., 1985, pp. 3278-97. 
Zhenguo Gu ( $M$ '93) received BSEE, 1982 from Tsinghua University, China, MSEE, 1990 and PhDEE, 1993 from University of Wisconsin-Madison. He has worked as an engineer or researcher in industries, research institutes, and universities for many years and accomplished many projects and papers in different fields, such as robotics,

power electronics, neural networks, signal processing and telecommunications. $\mathrm{He}$ is currently with $\mathrm{Oak}$ Ridge National Laboratory and working on building distribution system simulators with functions of GUI and data visualization, and on applications of neural networks, fuzzy logic and signal processing to power systems.

D. Tom Rizy (SM'87) received a BSEE from the University of Virginia in 1976 and an MSEE from Virginia Polytechnic Institute and State University in . . . . . 1977. He has worked as a researcher and project manager at the Oak Ridge National Laboratory, Oak Ridge, Tennessee since 1978. His responsibilities have included conducting and

managing electric power systems research in the areas of demand-side management; distribution system protection, safety, and automation; adaptive transmission system protection; reliability-centered maintenance; geomagnetic storms; and more recently real-time power systems control. He is a coauthor of a book on the Athens Automation and Control Experiment, a largescale distribution automation field experiment sponsored by the U.S. Department of Energy which was completed in 1987. He served on the IEEE Standards Coordinating Committee on Dispersed Storage and Generation, SCC 23, which was formed in 1982 and was responsible for producing two IEEE recommended practices for utility interconnection of small wind systems and a test procedure for utility static power converters; and a guide for interfacing dispersed sources with the electric utility system. He is one of the recipents of a 1990 IEEE Prize Paper Award for a paper on adaptive transmission system protection.

\section{DISCLAIMER}

This report was prepared as an account of work sponsored by an agency of the United States Government. Neither the United States Government nor any agency thereof, nor any of their employees, makes any warranty, express or implied, or assumes any legal liability or responsibility for the accuracy, completeness, or usefulness of any information, apparatus, product, or process disclosed, or represents that its use would not infringe privately owned rights. Reference herein to any specific commercial product, process, or service by trade name, trademark, mant necessarily constitute or imply its endorsement, recommendation, or favoring by the United States Government or any agency thereof. The views and opinions of authors expressed herein do not necessarily state or reflect those of the United States Government or any agency thereof. 